\title{
Effect of transformer noise on the neurophysiology of SD rats
}

\author{
YOU ZOU $^{1 *}$, XING YI $^{1 *}$, JIAN-GONG ZHANG ${ }^{2}$, XING-FA LIU ${ }^{2}$, KUN YANG $^{1}$, \\ YONG-GANG KONG ${ }^{1}$, BO-KUI XIAO ${ }^{3}$, ZE-ZHANG TAO ${ }^{1,3}$ and SHI-MING CHEN ${ }^{1,3}$ \\ ${ }^{1}$ Department of Otolaryngology Head and Neck Surgery, Renmin Hospital of Wuhan University, Wuhan, Hubei 430060; \\ ${ }^{2}$ China Electric Power Research Institute, Wuhan, Hubei 430070; ${ }^{3}$ Otolaryngology Head and Neck Surgery Institute, \\ Medical School of Wuhan University, Wuhan, Hubei 430060, P.R. China
}

Received July 17, 2018; Accepted January 24, 2019

DOI: $10.3892 /$ etm.2019.7360

\begin{abstract}
Transformer noise is a type of environmental sound that causes discomfort to individuals. The aim of the present study was to determine the effect of relatively long-term periods of transformer noise on the behavior and neurophysiology of SD rats. A total of 90 healthy SD rats with normal hearing were randomly divided into two experimental groups (65 and $60 \mathrm{~dB}$ group) and a control group. The experimental groups were exposed to recorded transformer noise for 8 weeks (sound level limits: 65 or $60 \mathrm{~dB}$ ) and the control group was maintained under the same conditions without noise stimulation. Changes in physiological growth (weight tests), behavior (tail suspension and open field behavior tests) and neurophysiology (glutamate, $\gamma$-aminobutyric acid, dopamine, 5-hydroxytryptamine, the morphologies of hippocampi) following noise exposure were recorded and compared. The results revealed that rats exhibited normal physiological growth, with no significant difference between the experimental and control groups. Following noise exposure, no significant differences were observed in the results of behavioral experiments (tail suspension and open field behavior tests) between the experimental and control groups. In addition, there were no significant differences in glutamate, $\gamma$-aminobutyric acid, dopamine and 5-hydroxytryptamine levels or in the morphologies of hippocampi between groups. In conclusion, exposure to transformer noise with a sound level limit of $65 \mathrm{~dB}$ sound pressure level (SPL) or $60 \mathrm{~dB}$ SPL (spectral range, 100-800 Hz) for 8 weeks (10 h/day) had no significant impact on the behavior and neurophysiology of SD rats.
\end{abstract}

Correspondence to: Professor Shi-Ming Chen, Department of Otolaryngology Head and Neck Surgery, Renmin Hospital of Wuhan University, 238 Jie-Fang Road, Wuhan, Hubei 430060, P.R. China E-mail: shimingchen0468@163.com

*Contributed equally

Key words: transformer noise, Sprague-Dawley rats, neurophysiology, behavior, hippocampus, neuron, synaptic morphology

\section{Introduction}

Transformers comprise the main equipment in converter stations, particularly in urban areas; they are also one of the main sources of noise from these locations (1). Major electric power research institutes worldwide are committed to the reasonable control of transformer noise in order to reduce public resistance to substation installation. However, complaints regarding transformer noise have not reduced in recent years (2). Transformer noise may cause discomfort as many individuals exhibit a low degree of tolerance for low-frequency noise at night (3). The A-weighted sound level used for fundamental sound measurement sometimes does not reflect the actual experience of individuals, particularly in relation to low-frequency noise (4-6). Therefore, environmental noise, particularly transformer noise, remains a prominent complaint despite extensive efforts to reduce and optimize transformer noise in converter stations. These complaints usually relate to the impact of noise on neurophysiological characteristics, including endocrine, mental or emotional conditions and sleep (7).

Transformer noise falls in the category of low-intensity and low-frequency noise $(8,9)$. The frequency of sound generated by transformers under normal operation is primarily concentrated in the $50-800 \mathrm{~Hz}$ range, while the intensity of the sound transmitted to surroundings is typically $<70 \mathrm{~dB}(10,11)$. To date, studies on noise have focused on short exposure time (generally no more than 8 weeks) and high intensity noise, including $120 \mathrm{~dB}$ (12), $118.9 \mathrm{~dB}(13), 100 \mathrm{~dB}(14), 126 \mathrm{~dB}(15)$ and $75 \mathrm{~dB}(16)$. In addition, Liu et al (17) demonstrated that a short-term period (35 days) of exposure to transformer noise with 60 and $65 \mathrm{~dB}$ had no effect on neurotransmitters and nervous tissue in the hippocampus of Sprague-Dawley (SD) rats. To the best of our knowledge, the effects of noise at $<70 \mathrm{~dB}$ (as applicable to transformer noise), over a relatively long-term period ( $>8$ weeks) have not yet been reported.

In order to determine whether exposure to a relatively long-term period ( 8 weeks) and low intensity $(60 / 65 \mathrm{~dB})$ of transformer noise has an impact on behavior and neurophysiological functions in experimental animals, SD rats were utilized in the current study. Rats were exposed to transformer noise recorded from residential areas and the resulting behavioral and neurophysiological effects were assessed. 


\section{Materials and methods}

Establishment of sound insulation environment. To simulate the transformer noise environment, an experimental apparatus was designed in the current study with good sound insulation (Fig. 1). The apparatus included a $2 \times 2 \times 2 \mathrm{~m}$ acoustic absorption cube with soundproof walls, in which the background noise was not higher than $35 \mathrm{~dB}$ after closing the door. The relative position of the sound source and sound insulation was adjusted so that noise distribution inside the device was uniform. The measurement of sound level meter (model, AWA 6291; Hangzhou Aihua Instruments Co., Ltd., China; www. hzaihua.com) revealed that noise intensity inside the device was no more than $3 \mathrm{~dB}$.

Animals and test groups. Ninety (45 male and 45 female) 6-week-old healthy adult SD rats (weight, 120-180 g) with similar behavioral index scores, good auricle reflex sensitivity and no middle ear infection were obtained from the Experimental Animal Research Center of Hubei Province (Wuhan, China). Animals were fed in an air-conditioned room (constant temperature, $22 \pm 2^{\circ} \mathrm{C}$; humidity, $50-60 \%$ ) with background noise $<35 \mathrm{~dB}$. Rats were housed in an artificial constant environment (12 h light/dark cycle, 08:00-20:00) with free access to food and water. Rats were then randomly divided into two experimental groups (65 and $60 \mathrm{~dB}$ group) and a control group (group $\mathrm{C}$; each, $\mathrm{n}=30$ per group; 1:1 male to female ratio in each group). The experimental groups were exposed to recorded transformer noise (sound level limits: 65 or $60 \mathrm{~dB}$ ). The control group was subjected to the same feeding conditions, but did not receive noise stimulation. The feeding and associated experiments were performed at the Center for Animal Experiments of Renmin Hospital of Wuhan University. All experiments were approved by the Committee on Ethics of Animal Experiments of Renmin Hospital of Wuhan University (Wuhan, China) and performed in compliance with the Guide for the Care and Use of Laboratory Animals from the National Institutes of Health (NIH publication no. 85-23, revised 1996).

Recording and exposure of transformer noise. The noise sample for the current study was obtained from a substation transformer (model DFPS-1000000/1000; rated capacity, 1,000 MVA; rated frequency, $50 \mathrm{~Hz}$; cooling method, oil forced air forced; TBEA Co., Ltd., Xinjiang, China). The sound sample collection point was $1.5 \mathrm{~m}$ above the ground and $1 \mathrm{~m}$ away from the transformer tank wall beside the side of the fan and the recording was taken when the transformer was working normally. An artificial head with binaural signal acquisition systems was used for collecting sounds and the recordings were analyzed using the ArtemiS 10.0 noise signal analysis software (Head Acoustics GmbH, Herzongenrath, Germany). The upper boundary of the recordings was $\sim 76 \mathrm{~dB}(\mathrm{~A})$, with a dominant frequency of 100-800 Hz. Prior to the experiment, recordings were treated using a power amplifier (Model, SWA100; BSWA Technology Co., Ltd., China, www.bswa. com.cn) to adjust the upper boundary to $65 \mathrm{~dB}(\mathrm{~A})$ or $60 \mathrm{~dB}(\mathrm{~A})$. The playback apparatus used was the dodecahedron speaker (Model, OS003; Beijing Sound Reputation Technology Co., Ltd., China). The sound environment distribution status in the sound arrester was detected using a simple sound level meter (Model, AWA 6291; Hangzhou Aihua Instruments Co., Ltd., Hangzhou, China) prior to playing the sound, in order to lower the difference due to the rats' activity to $3 \mathrm{~dB}$ by adjusting the relative position of the sound device. The sound level varied from $65 \pm 3$ or $60 \pm 3 \mathrm{~dB}$. Experimental groups were continuously exposed to the recorded noises for $10 \mathrm{~h} /$ day (from 22:00 to 8:00) for 8 weeks $(12,18)$. Each group had free access to food and water. The water bottles were filled twice daily. The drinking, diets and activities of the rats were observed daily, and the rats were weighed once per week.

Tail suspension test. After exposure to noise for 56 days, 10 randomly selected rats from each group were used to conduct the tail suspension test. The rat-tail suspension test device was made according to internationally established methods (16). Each rat was observed for 6 min and indices including struggle indicators (struggle amplitude of mouse head), total immobility time and longest immobility time within $6 \mathrm{~min}$, were recorded.

Open field test. After exposure to noise for 56 days, 10 randomly selected rats from each group were performed open field test. An open field with a size of 120x90x35 cm was created according to previous literature (17). The walls and floor were black. The floor was split into 9 rectangles (each, $40 \times 30 \mathrm{~cm}$ ) and the middle rectangle was defined as the center. The test was performed in a quiet, light and temperature-suitable room (constant temperature, $22 \pm 2^{\circ} \mathrm{C}$ ) between 8:00 and 12:00. Exploratory behavior of the rats in the open field was recorded and analyzed using the animal behavior tracking system (EthoVision 3.0; Noldus Information Technology bv, Wageningen, The Netherlands). Indicators of spontaneous motor activity, including total distance travelled, average speed, time spent in the central cell, rearing frequency (rat 'stands up' on its hind legs with the forelegs off the ground, regardless of the standing time) and the number of fecal pellets, were assessed over a $10 \mathrm{~min}$ period.

Detection of neurotransmitter content in the hippocampus. Following exposure to the recorded noises for $10 \mathrm{~h} /$ day for 8 weeks, 6 randomly selected rats from each group were anesthetized with isoflurane (Sumitomo Dainippon Pharma Co., Ltd., Osaka, Japan; 1 1/min $\mathrm{O}_{2}$ flow rate) at $4 \%$ for induction and $1.5-2 \%$ for maintenance. The anesthetized rats were then decapitated. Fresh hippocampal tissues were removed immediately. Following homogenization, High Performance Liquid Chromatography (HPLC) was performed for the quantitative analysis of the amino acid neurotransmitters glutamate (Glu) and $\gamma$-aminobutyric acid (GABA), and the monoamine neurotransmitters dopamine (DA) and 5-hydroxytryptamine (5-HT) in the hippocampus. The chromatographic conditions were: Chromatographic column from Hypersil ODS-3 4.6x250 mm, $5 \mu \mathrm{m}$ (GL Sciences, Inc., Tokyo, Japan); column temperature, $40^{\circ} \mathrm{C}$; mobile phase, potassium dihydrogen phosphate $(0.1 \mathrm{~mol} / \mathrm{l}, \mathrm{pH} 6.0)$ : methanol: acetonitrile at $6: 3: 1$; flow velocity, $1.0 \mathrm{ml} / \mathrm{min}$; emission wavelength, $455 \mathrm{~nm}$; and excitation wavelength, $340 \mathrm{~nm}$. For the hippocampus samples, $0.5 \mathrm{ml}$ samples were taken and added into tubes. Subsequently, $1 \mathrm{ml}$ of perchlorate was added after homogenization, then 


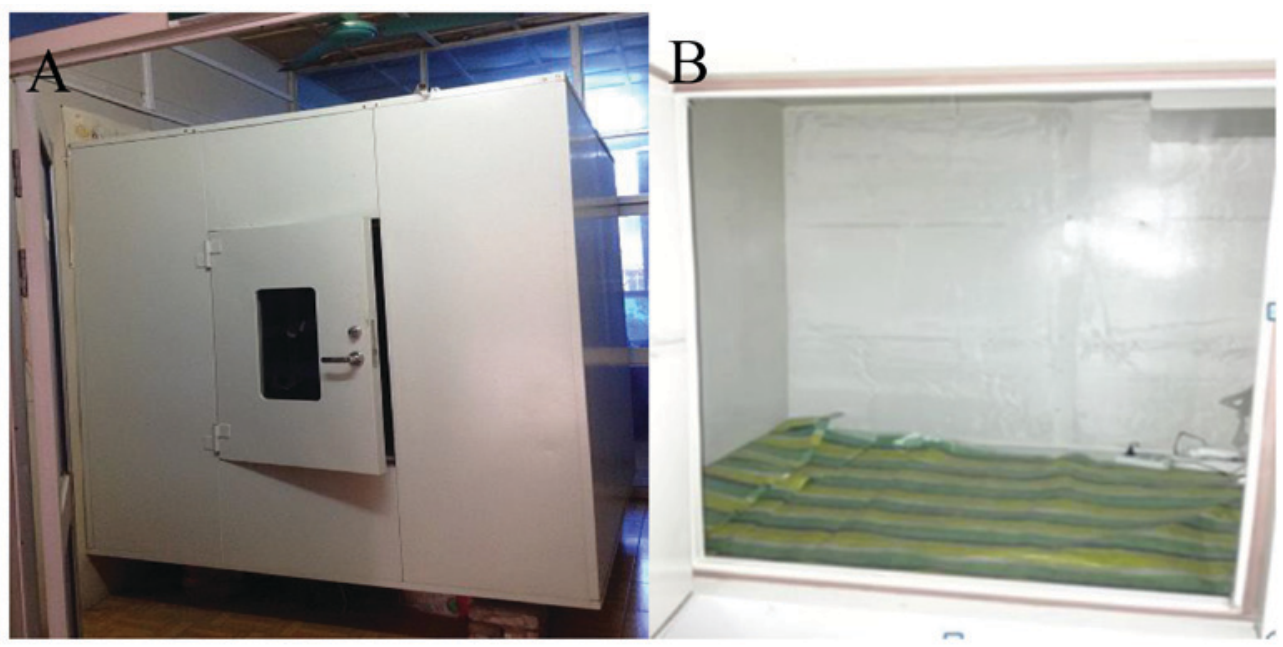

Figure 1. Sound-insulating experimental device: (A) outside and (B) inside.

Male

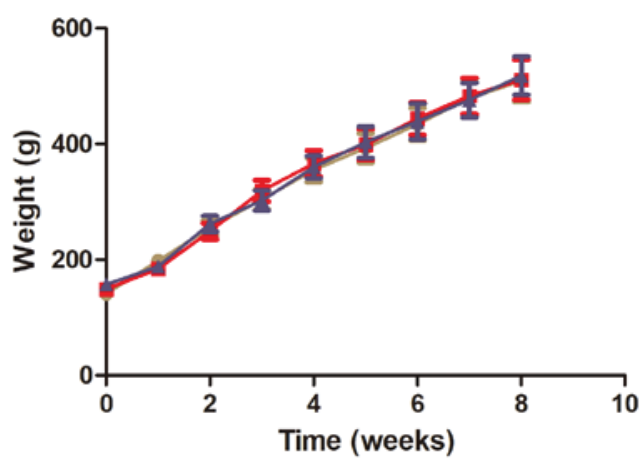

Female

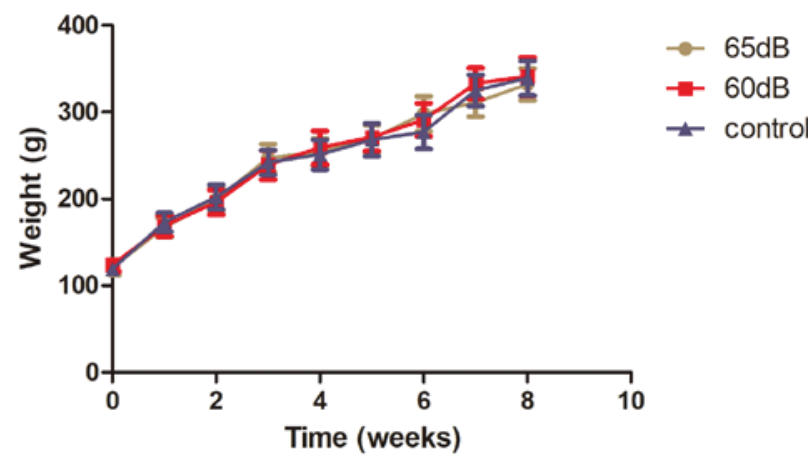

Figure 2. Animal weight changes (separated by sex) under exposure to noise. No significant differences between groups were identified.

centrifuged at $1,248 \times \mathrm{g}$ at $4^{\circ} \mathrm{C}$ for $15 \mathrm{~min}$. The supernatant was then transferred into another clean tube at $40^{\circ} \mathrm{C}, 100 \mu \mathrm{l}$ mobile phase was used for constant volume of the residue, followed by an injection of $20 \mu l$.

Transmission electron microscopy (TEM). Following exposure to the recorded noises for $10 \mathrm{~h}$ /day for 8 weeks, 4 randomly selected rats from each group were anesthetized with isoflurane $\left(1 \mathrm{l} / \mathrm{min}_{2}\right.$ flow rate) at $4 \%$ for induction and $1.5-2 \%$ for maintenance, where temperature was monitored with a rectal probe and maintained at $37.5 \pm 1^{\circ} \mathrm{C}$ using a non-electrical heating pad. The remaining 60 rats were used for further experiments not presented in the current study. SD rats were then perfused (via a transcardial approach) with $0.9 \%$ saline (Sinopharm Chemical Reagent Co., Ltd., Shanghai, China) and $2.5 \%$ glutaraldehyde (Sinopharm Chemical Reagent Co., Ltd.). Following euthanasia, hippocampal tissues were removed. Tissues were fixed by immersion in $2 \%$ glutaraldehyde at $4^{\circ} \mathrm{C}$ for $2 \mathrm{~h}$. Following rinsing in 0.1 M PBS (Jinuo Biomedical Technology Co., Ltd., Hangzhou, China; http://www.genom.com.cn/), the specimens were post-fixed in $1 \%$ OsO4 (Sinopharm Chemical Reagent Co., Ltd.) at $4^{\circ} \mathrm{C}$ for $1 \mathrm{~h}$, dehydrated in graded concentrations of acetone (Sinopharm Chemical Reagent Co., Ltd.) and embedded in a mixture of Epon and Araldite (Electron
Microscopy Sciences, Hatfield, PA, USA). Semithin sections, at $1 \mathrm{~mm}$ thickness, were stained with toluidine blue (Sinopharm Chemical Reagent Co., Ltd.) at $37^{\circ} \mathrm{C}$ for $30 \mathrm{sec}$. Ultrathin sections were cut to a thickness of $70 \mathrm{~nm}$, stained with lead citrate and uranyl acetate (Sigma-Aldrich; Merck KGaA, Darmstadt, Germany) at $37^{\circ} \mathrm{C}$ for $15 \mathrm{~min}$. Cell morphology and the apoptosis of hippocampal neurons were assessed via TEM (model, HT7800; Hitachi Ltd., Tokyo, Japan), with focus on the morphological changes of neuronal nuclei and synapses. The apoptotic neurons were condensed and had clumped chromatin with fragmentation of the nuclear membrane.

Statistical analysis. SPSS 18.0 (SPSS, Inc., Chicago, IL, USA) was used for all statistical analyses. All values were expressed as the mean \pm standard deviation. Statistical analyses were performed by one-way analysis of variance followed by the Student-Newman-Keuls post-hoc test. Homogeneity of variance was evaluated using the Levene's test. $\mathrm{P}<0.05$ was considered to indicate a statistically significant difference.

\section{Results}

General condition. Under exposure to transformer noise for $10 \mathrm{~h} /$ day, SD rats were generally in a healthy condition, 
A

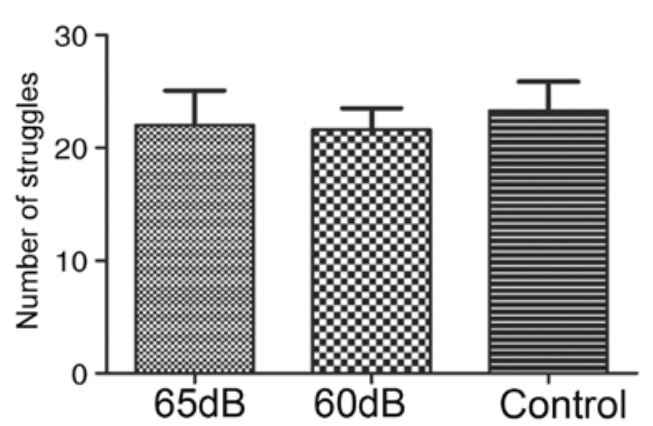

B

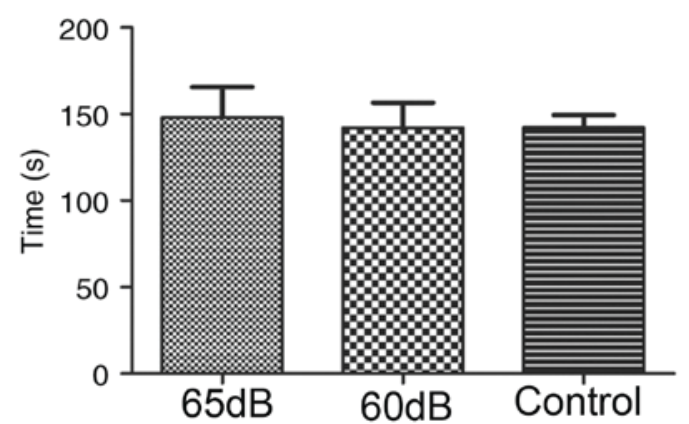

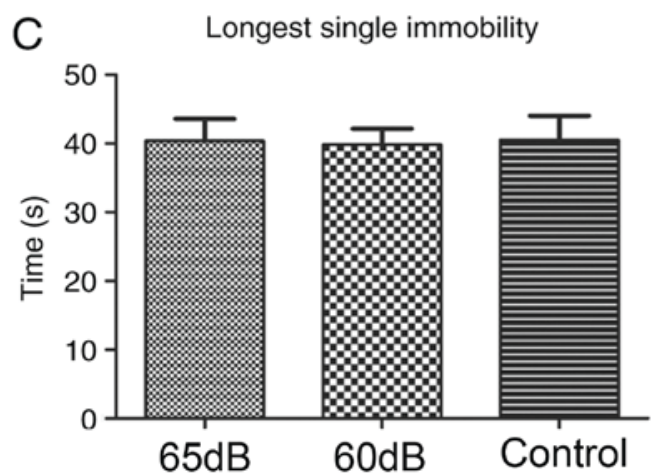

Figure 3. Tail suspension test indices following noise exposure. No significant difference in (A) struggle indicators (struggle amplitude of rat head), (B) total immobility time and (C) longest immobility time in $6 \mathrm{~min}$ between the experimental groups and the control group were identified.

as demonstrated by normal eating and drinking. The body weights of the animals in each group were recorded once a week from the first day of exposure and the trends between the groups were subsequently compared. During the 56 days of noise exposure, the body weight of rats in each group reflected normal physiological growth. There were no significant differences among the three groups (Fig. 2).

Tail suspension test. Following exposure to noise for 56 days, struggle indicators (struggle amplitude of mouse head), total immobility time and longest immobility time within 6 min were recorded. The results revealed no significant differences among the three groups (Fig. 3).

Open field test. Following exposure to noise for 56 days, the total distance travelled, average speed, residence time in the central cell, rearing frequency and defecation in $10 \mathrm{~min}$ were recorded (Fig. 4). No significant differences were identified among the three groups.

Amino acid neurotransmitters. HPLC was performed to analyze changes in Glu and GABA in hippocampal tissue. Fig. 5 presents a standard chromatogram (Fig. 5A) and a sample chromatogram of the amino acid neurotransmitters (Fig. 5B), as well as measurements of Glu and GABA content (Fig. 5C). There were no significant differences among the three groups.

Quantitative analysis of monoamine neurotransmitters. The changes in DA and 5-HT content in hippocampal tissue were analyzed via HPLC. Fig. 6 presents standard chromatograms
(Fig. 6A and B) and a sample chromatogram (Fig. 6C) of monoamine neurotransmitters, as well as the content of DA and 5-HT content (Fig. 6D). No significant differences were identified between groups.

Morphological observation of hippocampal neurons. TEM was performed to observe changes in the neuronal nuclei and synapses. Following exposure to noise for 8 weeks, hippocampal tissues from 4 randomly selected rats in each group were extracted and the morphological structure of the hippocampal neurons was observed using TEM. As presented in Fig. 7A-C, neuronal nuclei had a regular oval shape, were uniformly stained and exhibited a clear nuclear membrane structure, normal overall cell morphology and normal mitochondrial morphology in 65, $60 \mathrm{~dB}$ and control group. Neuronal morphology in all sections appeared similar. Furthermore, no marked morphological differences were identified among the groups. As presented in Fig. 7D-F, cells in the 65, $60 \mathrm{~dB}$ and control group exhibited a clear synaptic cleft and normal synaptic vesicle aggregation without edema. There were no marked differences among the three groups. (Fig. 7).

\section{Discussion}

Transformers are a prominent source of noise from power supporting systems in urban areas. Transformer noise is generated by cooling system fans and pumps, as well as mechanical movement caused by the operation of the machine and vibrations due to electromagnetic changes $(19,20)$. Generally, noise is considered a harmful physical stimulation, which can affect the growth and development of rats, impeding weight gain (21). 

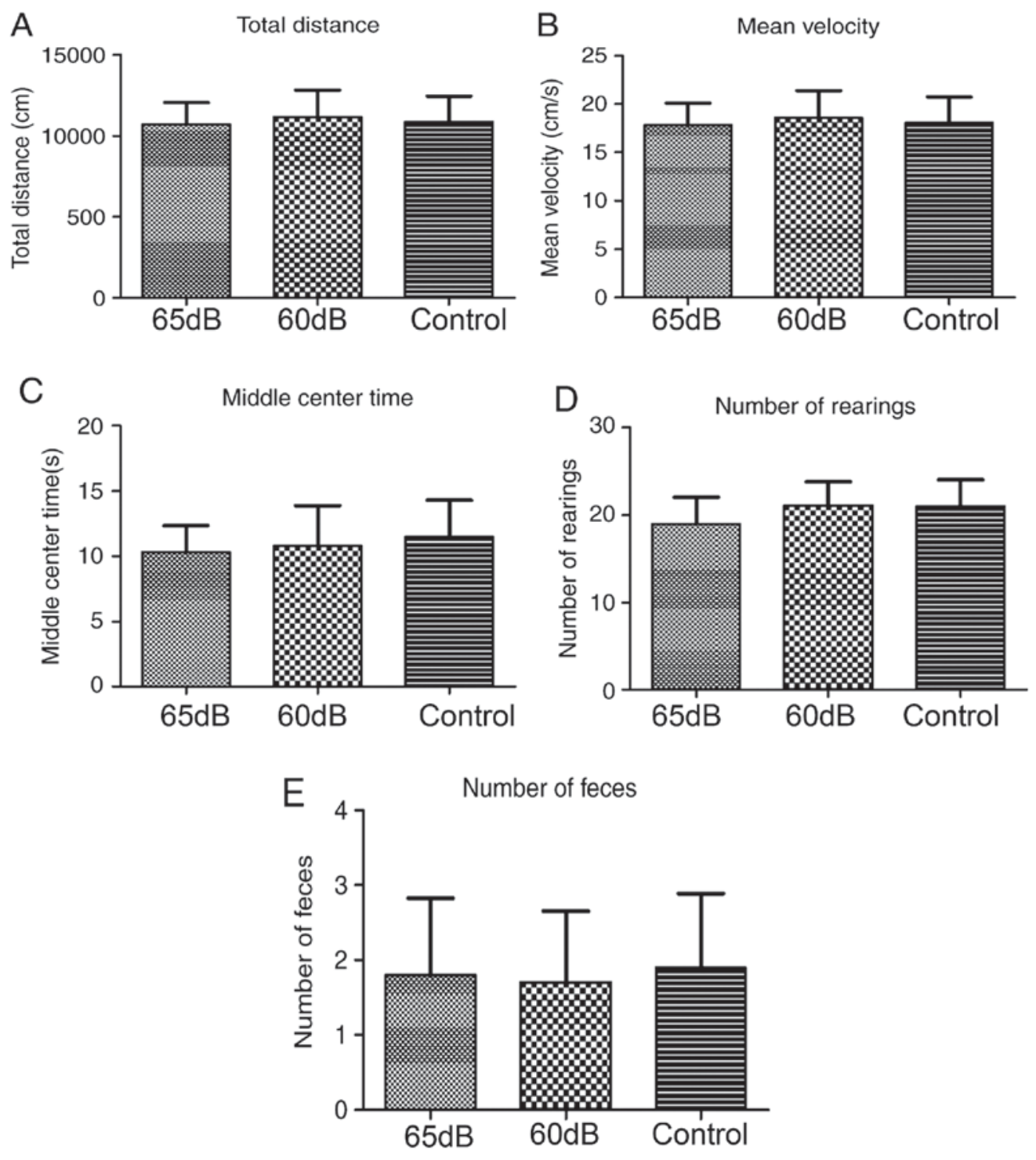

Figure 4. Comparison of open field test indices following noise exposure. No significant difference was identified in (A) the total distance travelled, (B) average speed, (C) residence time in the central cell, (D) rearing frequency and (E) defecation in 10 min between the experimental and control groups.

Exposure to noise pollution for a certain period of time and intensity may change the balance of energy metabolism, which would cause weight change $(22,23)$. The current study observed no significant differences in weight gain or food intake between experimental and control groups, indicating that $65-$ or $60-\mathrm{dB}$ sound pressure level (SPL) transformer noise exposure for 8 weeks had no significant influence on the growth of SD rats.

The tail suspension test is commonly employed to assess overall the physical strength, endurance and mental condition of animals in medical research (24). In the inverted position, the immobility time of animals reflects a state of 'behavioral despair' $(25,26)$. In the current study, no significant differences were identified in immobility time (total immobility and longest immobility time) among the three groups, indicating that exposure to transformer noise did not impact the endurance and mental condition of the rats. This may be due to the intensity of noise or the time of exposure being insufficient to produce measurable changes.

The open-field test is a classical behavioral experiment used to assess locomotor activity and anxiety in animals.
It is often performed to elucidate behavioral changes more comprehensively (27). In the present study, the three groups exhibited similar total travel distance and average speed, indicating that noise exposure for 8 weeks did not change the exploratory behavior and movement of the experimental group, which was consistent with the results of the tail suspension test. The central cell residence time of rats increased slightly as the intensity of sound noise exposure decreased; however, these differences were not statistically significant. However, there were no significant differences among the three groups, indicating that noise exposure did not affect the cognitive ability of SD rats. Furthermore, there were no significant differences in the upright times of the test and control animals, indicating that noise exposure did not impact on SD rat behavior. It was also demonstrated that the amount of feces in the experimental group was lower than that of the control group, indicating that transformer noise had no significant effect on the degree of tension in SD rats.

Epidemiological and experimental studies have revealed that noise can affect neurobehavioral function, causing 

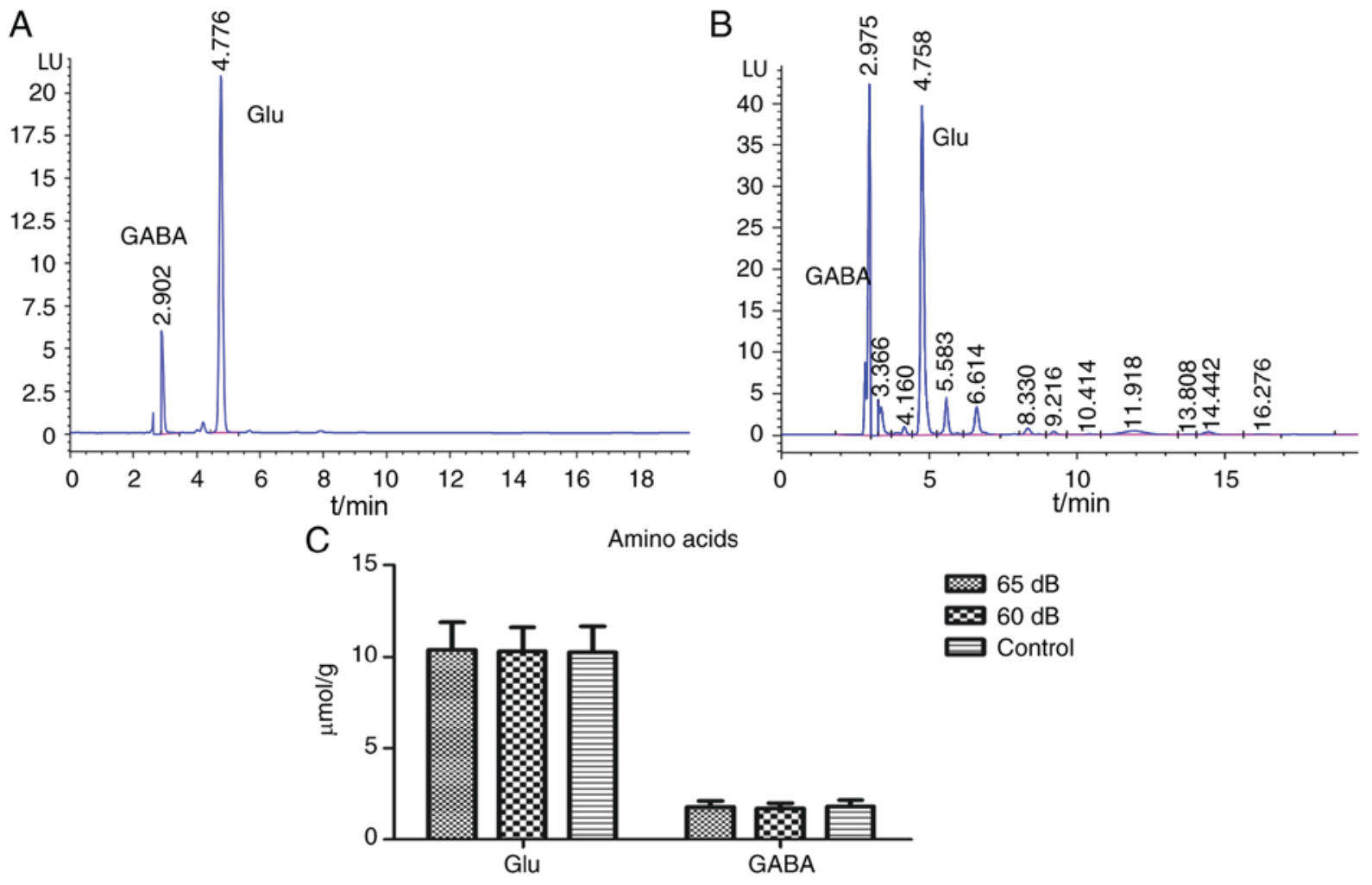

Figure 5. Comparison of amino acid neurotransmitter content in hippocampal tissue following exposure to noise for 56 days. (A) Standard chromatogram of amino acid neurotransmitters. (B) Chromatogram of hippocampal extracts. (C) Bar graph of amino acid neurotransmitter content measured via high-performance liquid chromatography. There were no significant differences among the three groups. GABA, $\gamma$-aminobutyric acid; Glu, glutamate.

A

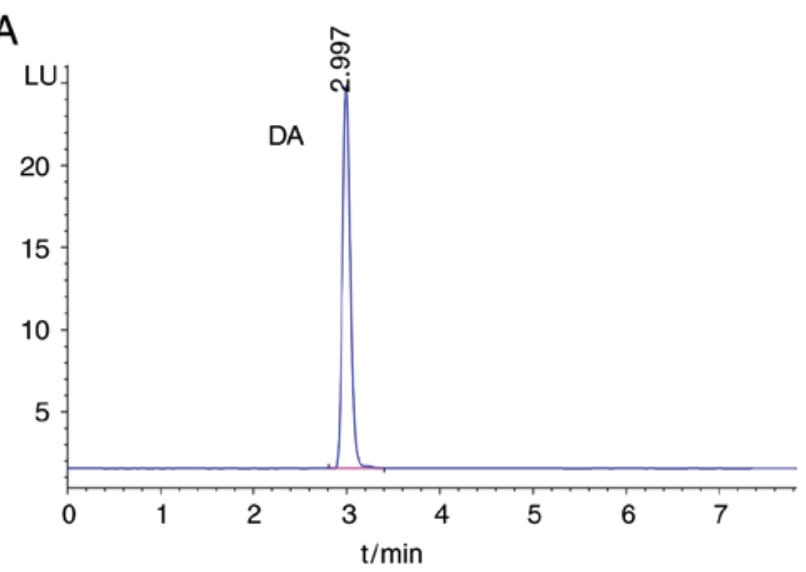

C

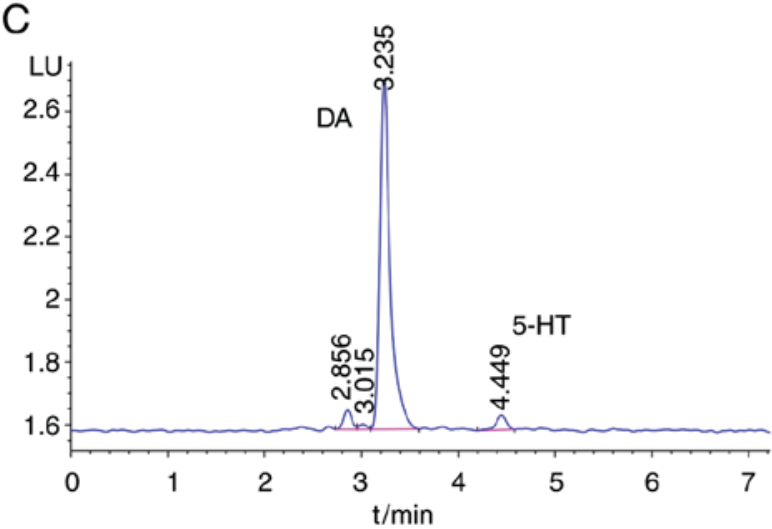

B

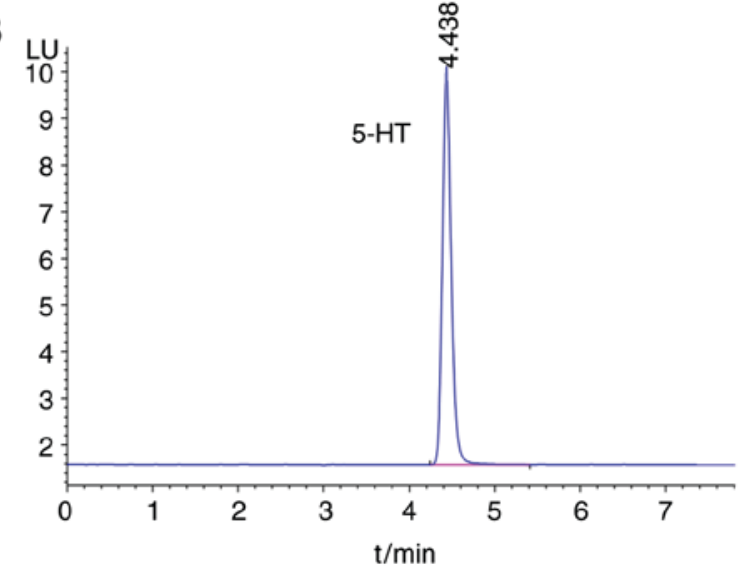

D

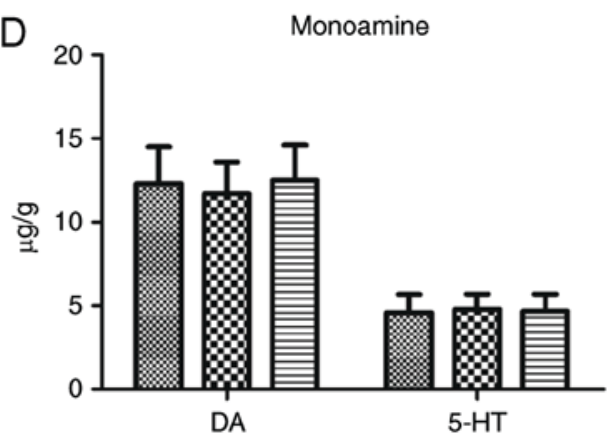

Figure 6. Comparison of monoamine neurotransmitter content in hippocampal tissue following exposure to noise for 56 days. (A) Standard chromatogram of DA. (B) Standard chromatogram of 5-HT. (C) Chromatogram of hippocampal extracts. (D) Bar graph of monoamine neurotransmitter content measured via high-performance liquid chromatography. There were no significant differences among the three groups. DA, dopamine; 5-HT, 5-hydroxytryptamine. 

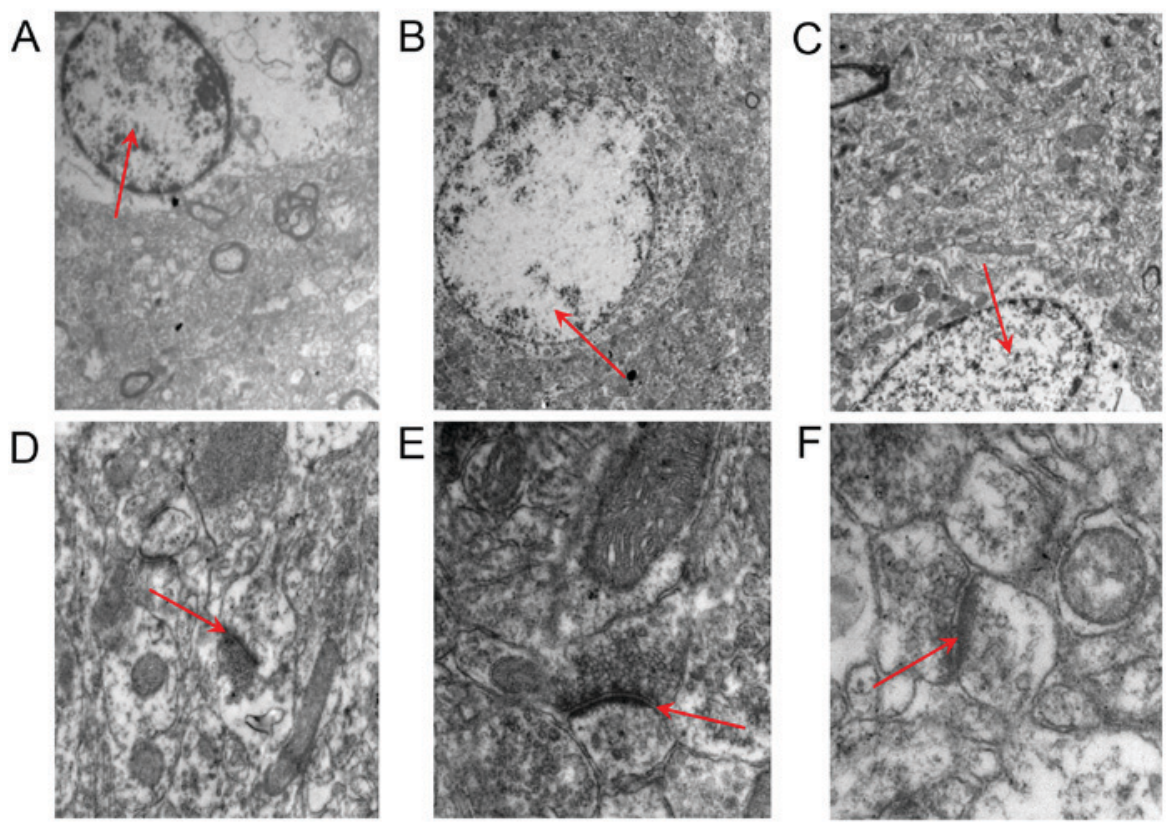

Figure 7. Comparison of morphology of hippocampal synaptic junctions and neurons following exposure to noise for 56 days. Morphology of the hippocampal neurons in the (A) $65 \mathrm{~dB},(\mathrm{~B}) 60 \mathrm{~dB}$ and (C) control groups. Neuronal nuclei were a regular oval shape, uniformly stained and exhibiting a clear nuclear membrane structure, normal morphology and normal mitochondrial morphology. No marked differences were identified among the three groups (magnification, x2,000; arrows indicate established neurons). Synaptic morphology of the hippocampus in (D) 65dB, (E) 60dB and (F) control groups. As indicated by the arrows, each slice exhibited a clear synaptic cleft and normal synaptic vesicle aggregation without edema. There were no marked differences among the three groups (magnification, x6,200; arrows indicate established synapses).

a series of effects including cognitive decline (28). The hippocampus is the primary functional area involved in learning, memory and emotional regulation (29). The level of amino acids in the hippocampus is closely associated with learning and memory (15,30-32). Noise exposure may cause environment changes involving chemicals including acetylcholine, amino acids, neurotransmitters, monoamine neurotransmitters, neuro-peptides and free radicals in the central nervous system, which are associated with cognitive abilities (33-36).

In the current study, the amino acid and monoamine neurotransmitter content in the hippocampus of rats exhibited no significant increase or decrease following exposure to 65 (A) or $60 \mathrm{~dB}(\mathrm{~A})$ transformer noise. The results of the amino acid (Glu and GABA) and monoamine neurotransmitter content (5-HT and DA) were consistent with those from a study by Liu et al (17). There was no significant difference in 5-HT content among the three groups; therefore, it may be hypothesized that transformer noise at the described intensity and exposure time may not result in depression (37). In addition, no marked damage or apoptosis was observed in the hippocampal neurons of the three groups. The results of the hippocampal neurons were also consistent with those from the study by Liu et al (17). This may be due to rats being at the peak of growth, with a very low chance of pathological change (38). These results indicated that a relatively long-term period of exposure to transformer noise did not affect the normal function and structure of the hippocampus in $\mathrm{SD}$ rats.

In summary, the results of the present study demonstrated that a relatively long-term period of exposure to transformer noise with a sound level limit of $65 \mathrm{~dB}$ SPL or $60 \mathrm{~dB}$ SPL (spectral range, 100-800 Hz) for 8 weeks $(10 \mathrm{~h} /$ day) had no significant impact on the neurophysiology of SD rats. Therefore, the current study hypothesizes that low-frequency and low-intensity noise, similar to transformer noise, may have no marked influence on the physiological function of the human body when exposed for a relatively long-term period. The results are worthy of further verification in a population with similar transformer noise exposure.

\section{Acknowledgements}

Not applicable.

\section{Funding}

The current study was supported by the Science and Technology Project of State Grid Corporation of China (grant no. GY71-13-057).

\section{Availability of data and materials}

The analyzed data sets generated during the study are available from the corresponding author on reasonable request.

\section{Authors' contributions}

SC conceived and designed the experiments of the current study. YZ performed the experiments and wrote the manuscript. XY performed the experiments and analyzed the data. JZ, XL, KY, YK, BX and ZT were responsible for data acquisition, analysis and interpretation. All the authors read and approved the final version of the manuscript. 


\section{Ethics approval and consent to participate}

All experiments were approved by the Committee on Ethics of Animal Experiments of Renmin Hospital of Wuhan University (Wuhan, China).

\section{Patient consent for publication}

Not applicable.

\section{Competing interests}

The authors declare that they have no competing interests.

\section{References}

1. Di GQ, Zhou XX and Chen XW: Annoyance response to low frequency noise with tonal components: A case study on transformer noise. Appl Acoust 91: 40-46, 2015.

2. Teoh C, Soh K, Zhou R, Tien D and Chan V: Active noise control of transformer noise. In: Proceedings of the International Conference on Energy Management and Power Delivery. IEEE, Singapore, 1998.

3. Waye KP, Clow A, Edwards S, Hucklebridge F and Rylander R: Effects of night-time low frequency noise on the cortisol response to awakening and subjectiv sleep quality. Life Sci 72 : 863-875, 2003.

4. Leventhall HG: Low frequency noise and annoyance. Noise Health 6: 59-72, 2004.

5. Kjellberg A, Tesarz M, Holmberg K and Landstrom U: Evaluation of frequency-weighted sound level measurements for prediction of low-frequency noise annoyance. Environ Int 23: 519-527, 1997.

6. Holmberg K, Landström U and Kjellberg A: Low frequency noise level variations and annoyance in working environments. J Low Freq Noise Vibrat Active Control 16: 81-87, 1997.

7. Muzet A: Environmental noise, sleep and health. Sleep Med Rev 11: 135-142, 2007.

8. Li Z and Di G: Reduce subjective annoyance from transformer noise by the method of sound adjustment. Acta Acust United Acustica 102: 452-461, 2016.

9. Olsen KN and Stevens CJ: Perceptual overestimation of rising intensity: Is stimulus continuity necessary? Perception 39: 695-704, 2010

10. Lau S: Code for Design of Sound Insulation of Civil Buildings GB 50118-2010 (Beijing: China Building Industry Press) 2010.

11. Yong W and Ji ZY: Impact of transformer noise on indoor residential environment and control techniques. Environ Monit Forewarning 2: 44-45, 2009.

12. Van Campen LE, Murphy WJ, Franks JR, Mathias PI and Toraason MA: Oxidative DNA damage is associated with intense noise exposure in the rat. Hear Res 164: 29-38, 2002.

13. Coppola CL, Enns RM and Grandin T: Noise in the animal shelter environment: Building design and the effects of daily noise exposure. J Appl Anim Welf Sci 9: 1-7, 2006.

14. Demirel R, Mollaoğlu H, Yeşilyurt H, Üçok K, Ayçiçek A, Akkaya M, Genç A, Uygur R and Doğan M: Noise induces oxidative stress in rat. Eur J Gen Med 6: 20-24, 2009.

15. Kraus KS, Mitra S, Jimenez Z, Hinduja S, Ding D, Jiang H, Gray L, Lobarinas E, Sun W and Salvi RJ: Noise trauma impairs neurogenesis in the rat hippocampus. Neuroscience 167: $1216-1226,2010$.

16. Di G, Zhou B and Lin Q: The effects of aircraft noise exposure on rat behavior and serum neurotransmitter expression. Noise Control Eng J 59: 514-518, 2011.

17. Liu XF, Tang YM, Zhou B, Liu ZH, Wan BQ, Zhang JG, Li W, Qu M and Tang LJ: Experimental research on effect of transformer noise exposure below $65 \mathrm{~dB}$ on the neurotransmitter and nervoustissue in hippocampus of SD rats. High Volt Eng 43: 2486-2495, 2017.

18. Yost WA, Koita N, Maslo R and Patel P: Dosimeter measures of sound exposure experienced by university students. J Acoust Soc America 120: 3163-3163, 2006.
19. Moses AJ: Measurement of magnetostriction and vibration with regard to transformer noise. IEEE Transact Magnet 10: 154-156, 1974.

20. Berglund B, Hassmén P and Job RFS: Sources and effects of low-frequency noise. J Acoust Soc Am 99: 2985-3002, 1996.

21. Smiley CS and Wilbanks WA: Some effects of noise exposure on early development in the albino rat. J Acoust Soc America 67 (Suppl 1): S58-S59, 1980.

22. Lasky RE and Williams AL: Noise and light exposures for extremely low birth weight newborns during their stay in the neonatal intensive care unit. Pediatrics 123: 540-546, 2009.

23. Michaud DS, Miller SM, Ferrarotto C, Keith SE, Bowers WJ, Kumarathsan P, Marro L and Trivedi A: Exposure to chronic noise and fractionated $\mathrm{X}$-ray radiation elicits biochemical changes and disrupts body weight gain in rat. Int J Radiat Biol 81: 299-307, 2005.

24. Yin C, Gou L, Liu Y, Yin X, Zhang L, Jia G and Zhuang X: Antidepressant-like effects of L-theanine in the forced swim and tail suspension tests in mice. Phytother Res 25: 1636-1639, 2011.

25. Steru L, Chermat R, Thierry B and Simon P: The tail suspension test: A new method for screening antidepressants in mice. Psychopharmacology 85: 367-370, 1985.

26. Barfield ET, Barry SM, Hodgin HB, Thompson BM, Allen SS and Grisel JE: Beta-endorphin mediates behavioral despair and the effect of ethanol on the tail suspension test in mice. Alcohol Clin Exp Res 34: 1066-1072, 2010.

27. Brenes Sáenz JC, Villagra OR and Fornaguera Trías J: Factor analysis of Forced swimming test, sucrose preference test and open field test on enriched, social and isolated reared rats. Behav Brain Res 169: 57-65, 2006.

28. van Kempen E, van Kamp I, Lebret E, Lammers J, Emmen H and Stansfeld S: Neurobehavioral effects of transportation noise in primary schoolchildren: A cross-sectional study. Environ Health 9: 25, 2010

29. Toyoda A, Iio W, Goto T, Koike H and Tsukahara T: Differential expression of genes encoding neurotrophic factors and their receptors along the septal-temporal axis of the rat hippocampus. Anim Sci J 85: 986-993, 2014.

30. Kim H, Lee MH, Chang HK, Lee TH, Lee HH, Shin MC, Shin MS, Won R, Shin HS and Kim CJ: Influence of prenatal noise and music on the spatial memory and neurogenesis in the hippocampus of developing rats. Brain Dev 28: 109-114, 2006.

31. Cheng L, Wang SH, Huang Y and Liao XM: The hippocampus may be more susceptible to environmental noise than the auditory cortex. Hear Res 333: 93-97, 2016.

32. Thiel CM, Müller CP, Huston JP and Schwarting RK: Auditory noise can prevent increased extracellular acetylcholine levels in the hippocampus in response to aversive stimulation. Brain Res 882: 112-119, 2000

33. Ravindran R, Rathinasamy SD, Samson J and Senthilvelan M: Noise-stress-induced brain neurotransmitter changes and the effect of Ocimum sanctum (Linn) treatment in albino rats. J Pharmacol Sci 98: 354-60, 2005.

34. Cui B, Wu M, She X and Liu H: Impulse noise exposure in rats causes cognitive deficits and changes in hippocampal neurotransmitter signaling and tau phosphorylation. Brain Res 1427: 35-43, 2012.

35. Gil-Loyzaga P, Vicente-Torres MA, Fernández-Mateos P, Arce A and Esquifino A: Piribedil affects dopamine turnover in cochleas stimulated by white noise. Hear Res 79: 178-182, 1994.

36. Hassanvand $\mathrm{T}$, Balooch $\mathrm{M}$, Azarnia $\mathrm{M}$ and Zardooz $\mathrm{H}$ : Alterations in dopamine related behavior of the offspring of pregnant Wistar rats exposed to noise pollution stress. Physiol Pharmacol 16: 79-85, 2012.

37. Van Praag HM: 5-HT-related, anxiety- and/or aggression-driven depression. Int Clin Psychopharmacol 9 (Suppl 1): S5-S6, 1994.

38. Turner CA, Clinton SM, Thompson RC, Watson SJ Jr and Akill H: Fibroblast growth factor-2 (FGF2) augmentation early in life alters hippocampal development and rescues the anxiety phenotype in vulnerable animals. Proc Natl Acad Sci USA 108: 8021-8025, 2011.

This work is licensed under a Creative Commons Attribution-NonCommercial-NoDerivatives 4.0 International (CC BY-NC-ND 4.0) License. 